\title{
Dynamic feedbacks among tree species traits, termites and an endangered mammal via deadwood turnover
}

\author{
Chao Guo ${ }^{1}$, Hans Cornelissen ${ }^{2}$, Bin $\mathrm{Tuo}^{1}$, Hang $\mathrm{Ci}^{1}$, and Enrong Yan ${ }^{1}$ \\ ${ }^{1}$ East China Normal University \\ ${ }^{2}$ VU University Amsterdam
}

July 27, 2020

\begin{abstract}
Biodiversity losses may impact ecosystem processes via food-webs. We hypothesized that time can modulate feedbacks among plant functional diversity, resource quality, prey and predator populations and litter turnover. Thereto we incubated coarse deadwood of 34 woody species, with and without (wood-feeding) termite access, in Chinese subtropical forests with and without (termite-feeding) pangolins. The results supported our hypothesis: in the first 12 months, termites amplified the positive linear relationship between \% wood mass loss and initial wood quality (along a wood economics spectrum, WES). In contrast, between 12 and 18 months, termite-mediated consumption, and associated wood mass loss, showed a humpback relation with the initial WES. This shift in termite preference of deadwood species along the WES indicated complementary food availability to termites through time, thereby promoting both termites and endangered pangolins. Thus, plant functional diversity through time can help to sustain keystone consumers, predators and their effects on carbon turnover.
\end{abstract}

\section{INTRODUCTION}

Biodiversity is declining dramatically as a result of habitat loss, unsustainable hunting and climate change, which may negatively affect ecosystem processes and functions (IP-BES Report 2019). Much research on terrestrial plant biodiversity has focused on how the loss of plant species or functional groups affects primary productivity (Hector et al.1999; Hooper \& Dukes 2004; Balvanera et al . 2006; Fargione et al. 2007), nutrient cycling and carbon flux (Hooper \& Vitousek 1998; Hättenschwiler 2005; Handa et al. 2014), e.g., through niche complementarity of plants or microbes. Within this biodiversity-ecosystem function debate, the emphasis has shifted from effects of taxonomic diversity or different coarse functional groups to effects of continuous variation and diversity in functional traits at the species level (Violle et al. 2007), i.e., functional diversity (Díaz et al. 2007; Handa et al. 2014). However, this work has hardly addressed how biodiversity affects ecosystem functions and services via multi-trophic interactions (but see Bascompte et al. 2003; Scherber et al. 2010). Those interactions are often hard to unravel, because, on the one hand, "top-down control" of predators on prey populations may affect nutrient flows in food webs (Srivastava et al. 2009) while on the other hand, the quality and availability of plant-based resources affect the population dynamics of consumers and their predators through "bottom-up control" (Haddad et al. 2009; Scherber et al. 2010). Moreover, multi-trophic interactions might prevail if key-stone of consumers or predators are involved, as ecosystems might be vulnerable to environmental changes that interfere with their population (Joneset al. 1997).

Plant functional diversity, via species' resource complementarity, may regulate the availability of basal food resources for consumers, and consequently higher trophic levels (Thakur \& Eisenhauer 2015). However, in natural ecosystems the resource supplied by plants might change over time, e.g., as a consequence of interspecific variation in plant phenology in the consumer food web or changes in dead matter quantity and quality in the detritivore food web. Green (plant) food webs can be structured by environmental changes at different 
time scales, e.g., seasonal, inter-annual and decadal (Voigt et al. 2003; McMeans et al. 2015). However, to our knowledge no previous studies have addressed how temporal variance in food availability across plant species could lead to temporal resource complementarity to higher trophic levels in brown (detritivore) food webs. Such temporal variance in food availability could occur, for instance, in deadwood of tree species of different initial trait composition and decomposing at different rates. In addition, in the opposite direction, higher predator densities can suppress populations of prey "top-down", which can induce interactive effects, or feedbacks, between predator densities and plant functional diversity on prey populations (Scherber et al. 2010; Haddad et al.2011). Such multitrophic feedbacks could also be affected by temporal variance (including complementarity) in quantity or quality of food supply, with potentially important consequences for ecosystem processes such as the turnover of dead plant matter. No previous theoretical or empirical studies have addressed this hypothesis.

Here we test this hypothesis in an intriguing system in warm-climate forests featuring trophic feedbacks between tree functional diversity, the dynamic resource (trait) diversity in the coarse deadwood derived from them, termites as their key-stone consumers and pangolins as the key-stone predators of the termites (Fig. 1). Pangolins are among the most endangered mammals in the world owing to poaching and illegal trafficking as well as deforestation; all eight Asian and African species range in status from "vulnerable" to "critically endangered" as a result (Corlett 2007; Wu \& Ma 2007; IUCN Red List of Threatened Species 2016). By consuming termites and controlling their populations, pangolins may affect forest carbon dynamics, as termites are the primary invertebrate deadwood decomposer (Wood \& Sands 1978; Ulyshen 2016), found to contribute for instance about half to deadwood turnover in southern China (Liu et al.2015). At the opposite end of the brown food web, deadwood quality, as determined by several wood traits, affects wood palatability to termites. Previous studies have shown that termites prefer to consume softer deadwood (Takamura 2001; Liu et al.2015). Therefore, the continuous availability of deadwood with favourable trait composition should help termite populations to thrive, thereby supporting the population of pangolins. However, changes in deadwood quality of each given tree species through the decay trajectory may affect the functional diversity dynamics of deadwood. How the feedbacks in this brown food web between pangolins, termites and deadwood traits affect wood decomposition rates across tree species, and how these feedbacks change over time, remains unknown.

Based on the theory above and our field observations in subtropical broad-leaved forests in China, we propose a new conceptual model that links natural temporal patterns in predator and detritivore populations and in decomposition with temporal patterns in functional traits across species (Fig. 1). It proposes that, after strong forest logging or typhoon impact, a set of functionally different woody species (ranging widely in initial wood quality) will provide a major food resource to the forest floor. Termites, being selective deadwood consumers, will first consume high-quality tree species, with low wood density, dry matter and lignin content, and high wood nutrient and cellulose content. Such a set of traits is associated with a resource-acquisitive growth strategy along a "wood economics spectrum" (WES; Baraloto et al. 2010; Freschet et al.2012; Pietsch et al. 2014; Zuo et al. 2018). Over time, after the high-quality wood has been mostly eaten (mainly by the termites), we predict that the initially medium-quality wood becomes more palatable owing to initial microbial activity and decomposition. Thus, the initially medium-quality wood (i.e., with intermediate WES values) should now be consumed preferentially by termites. This temporal pattern would imply that, in case of a broad range of initial wood qualities along the WES, i.e., high functional diversity, there will be a stable provisioning of sufficient food resource for termites to maintain their population through time. In turn, a larger and stable termite population may increase the density of pangolins. Taken together, we hypothesize that woody plant functional (trait) diversity might support a threatened mammal species at the top of the food chain. Simultaneously, the consumption of deadwood by termites should sustain deadwood turnover in a forest with high plant functional diversity (Fig. 1) by the same temporal mechanism of changing wood quality over time. Termite predation by pangolins might constrain the termite population size, thereby indirectly reducing forest-scale wood decomposition rate and enhancing its carbon storage. In an alternative scenario, where pangolins as the key predator are lost from the forest, e.g., through hunting, these multi-trophic feedbacks might change, and might alter the carbon budget of a forest, via increased turnover of deadwood 
by termites, again mediated by deadwood functional diversity.

We put our conceptual framework to the experimental test by comparing deadwood traits and decomposition rates of 34 tree species, termite abundance and their contributions to decomposition rates in two subtropical evergreen broad-leaved forest sites in Eastern China: one with and one without Chinese pangolins (Manis pentadactyla). In each of these two forest sites we carried out an identical 18-month coarse deadwood decomposition experiment with 34 woody species, with and without termite exclusion.

\section{MATERIALS AND METHODS}

\section{Study systems}

We conducted this study in two sites in Zhejiang Province, East China: i) Tiantong National Forest Park (TT) (29 $\left.52^{\prime} \mathrm{N}, 121 \mathrm{deg} 39^{\prime} \mathrm{E}\right)$, which has a subtropical monsoon climate. The dominant vegetation is subtropical evergreen broad-leaved forest. ii) Putuo island (PT) (29deg97'N, 121deg38'E), in the Zhoushan archipelago at $6.5 \mathrm{~km}$ from the mainland, has a marine subtropical monsoon climate. The island supports evergreen broadleaved forests dominated by Cyclobalanopsis glauca and mixed evergreen-deciduous broad-leaved forests. On the mainland, Chinese pangolins (Manis pentadactyla) are critically endangered and no pangolin evidence was observed TT during the two study years. In contrast, in PT the Chinese pangolin population is thriving because of conservation efforts and the island's isolation from the mainland. In both sites, termites (mainly Coptotermes formosanus, Odontotermes formosanus, Reticulitermes chinensis, Reticulitermes speratus ) are the major deadwood decomposers (Yi et al. 2006). Table 1 has further data for abiotic and biotic features of both sites (see also Yanet al . 2006).

\section{Tree species sampling and wood decomposition experiment}

From October to November 2017, we selected 32 important and representative woody species in TT and $\mathrm{PT}$, and 2 island endemic woody species in PT (Pittosporum tobira, Eurya emarginata). The species set included 7 evergreen trees and 11 evergreen shrubs, 7 deciduous trees and 4 deciduous shrubs, 4 conifer trees and 1 monocot tree (bamboo) (full species names see Supp. Mat. Table S1). Here "shrubs" included both short stature, single-stem understorey trees and multi-stemmed shrubs from exposed habitats. In total, 147 individual healthy adult trees/shrubs were selected, i.e., 34 species x 3 replications (but 6 replications for shrubs). We chain-sawed those trees/shrubs and collected wood samples as 20-cm long stem sections of 5 +$0.5 \mathrm{~cm}$ diameter so as to control for size effects on decomposition. Adjacent to each end a 2 -cm thick disk was sawn out for analyses of initial wood traits. Here we used the healthy living trees standardizing the initial, undegraded phase for all samples, thereby avoiding complications of prior pathogen attack having altered wood quality and providing a direct link to the functional traits of living trees (Cornelissen et al. 2012). Moreover, in the forests studied typhoon-induced wind-throw as well as logging is very common, so a large fraction of the trees is still alive when downed. We therefore consider these wood samples as representative of deadwood going into the decomposition process.

We used $25 \times 15 \mathrm{~cm}$ litter-bags differing in mesh size to assess the termite contribution to litter decomposition: (1) termite exclusion treatment, enclosed stainless steel litter-bags with $0.05 \mathrm{~mm}$ mesh; (2) termite access treatment, nylon litter-bags with $4 \mathrm{~mm}$ mesh. Each fresh wood sample was weighed immediately after cutting (see below for measurement of water content), and sealed into a litter-bag. In total, 1224 litter-bag samples were produced, i.e., 34 tree species x 2 treatments $\mathrm{x} 3$ replications (plots) x 2 incubation sites $\mathrm{x} 3$ harvest times.

In December 2017, we established three $20 \mathrm{~m}$ x $30 \mathrm{~m}$ replicate plots in PT and TT, respectively. Plots within each site had broadly similar slope, altitude, soil type, litter substrate and forest structure and composition and plots within a site were $10 \mathrm{~m}$ apart to minimize interference between them. We started litter-bag incubation in December 2017, when termite activity and wood consumption was low because of low temperatures. The litter-bags of each species were pinned onto the forest floor in their respective subplots within each of the three replicate plots randomly, and each species' replicate had three litter-bags per treatment in each subplot, i.e., one for each harvest. The distances between subplots were approx. $2 \mathrm{~m}$. 
Litter-bags were harvested after 6 months (July 2018), 12 months (Dec. 2018) and 18 months (July 2019).

We transported the litter-bags to the laboratory where we carefully removed extraneous materials and soil from the deadwood remains within a few days. Each wood sample was put in a large tray with tall enough edges for the termites not to crawl out. We cut the sample into small pieces and cleaned mud and sand (brought in by termites) with a brush carefully. We then collected the termites from the tray and counted them. All pieces of the wood sample were then oven-dried at $75 \operatorname{deg} \mathrm{C}$ to constant mass and weighed (dry mass).

\section{Wood trait measurements}

For measurement of initial wood traits considered relevant to the WES and to decomposition, each 2-cm sub-sample was stored in a sealed plastic bag immediately after collection in the field, and kept cool until processing. Within $12 \mathrm{~h}$, after the bark had been removed, a sub-sample was cut from each disk to obtain fresh mass initial volume using Archimedes' principle of water displacement (Williamson \& Wiemann 2010).

All wood sub-samples were dried at $75 \mathrm{degC}$ for $72 \mathrm{~h}$ to determine dry mass. Initial wood density $\left(\mathrm{g}^{*} \mathrm{~cm}^{-3}\right)$ was calculated as dry mass per volume. Initial water content, i.e., (fresh mass-dry mass)/ fresh mass, was used to calculate the initial dry mass of the litter-bag samples. Initial wood sub-samples were ground in a laboratory mill and passed through a $0.15 \mathrm{~mm}$ sieve. Thereafter, $0.2 \mathrm{~g}$ sub-samples were digested using concentrated $\mathrm{H}_{2} \mathrm{SO}_{4}$ to determine $\mathrm{N}$ and $\mathrm{P}$ concentrations on an infrared spectrophotometer (Smartchem 200, Alliance, France). Lignin content was determined by acidolysis -titration method, and cellulose content was determined by anthrone-sulfuric acid colorimetry (Poorter \& Villar 1997).

\section{Termite and pangolin abundance measurements}

To quantify the termite abundance in situ, we sampled the termites in the experimental plots of PT and TT in July 2018 and July 2019 adopting the (2000) transects method (Jones \& Eggleton 2000). Since the area of our plots was $30 \times 20 \mathrm{~m}$, we took our entire plot as belt transect, divided into ten $3 \times 2 \mathrm{~m}$ sections. In each section we counted the number of termites with an area meter (with $10 \mathrm{x} 10 \mathrm{~cm}$ grid), based on counts of $1 \times 1 \mathrm{~cm}$ grid cells across the diagonal. We scaled these data up to the plot and also examined all deadwood and leaf litter for termites to obtain total termite abundance of each plot. See above for termite counts in the harvested deadwood samples.

We determined the density of pangolins in PT by counting their mounds in each incubation plot, simultaneously, in three nearby plots of the same area but without wood samples.

\section{Calculation of wood mass loss rate}

Wood litter mass loss rate \% was calculated according to Eqn. 1:

\section{Hosted file}

image1.wmf available at https://authorea.com/users/346616/articles/472545-dynamic-feedbacksamong-tree-species-traits-termites-and-an-endangered-mammal-via-deadwood-turnover

(Eqn. 1)

where $M_{i}$ and $M_{f}$ are initial and final wood dry mass; when calculating the period-specific mass loss, $M_{i}$ is the final wood dry mass of the previous stage and $\mathrm{M}_{\mathrm{f}}$ is the final wood dry mass for this period.

The termite contribution decomposition was calculated as effect size (Borensteinet al. 2009) according to Eqn. 2, based on termite access and exclusion treatments described above.

(Eqn. 2)

\section{Statistical analysis}


To quantify the main axes of wood trait variation across species, a principal component analysis (PCA) was performed. The first axis (PC1), accounting for $55.9 \%$ of variance in litter quality, was strongly related to the contents of wood nutrients (nitrogen, phosphorus), cellulose and lignin, and wood density. We used the PC1 scores for the respective tree species to represent their position along the WES positions in the subsequent analyses. The second axis (PC2) was related to wood water content, accounting for $17.1 \%$ of variance (Fig. S1).

We also used PCA to quantify the community-level tree functional trait variability for forest plots in TT and PT. The PC1 of PT and TT accounted for $50.3 \%$ and $40.9 \%$ of trait variance, respectively and were strongly related to leaf resource economic traits (specific leaf area, nitrogen, phosphorus, mean leaf area) and wood density (Fig. S4). We used the community abundance-weighted mean (CWM) of WES, specific leaf area and wood density to compare differences of community functional identity between PT and TT sites by using Student's t-tests. To derive CWM of WES we multiplied the PC1 scores of each species with its relative abundance for a given community.

We used ANCOVA to determine the dependence of wood mass loss on specific independent variables. Using the wood (cumulative or period) mass loss $\%$ as the dependent variable, harvest time and termite presence/absence as the independent variables, and the WES value as covariate, separate ANCOVAs were performed for PT and TT respectively. To evaluate the relationships between (cumulative) mass loss \% (for termite access and exclusion treatments) and position along the WES separately for the different incubation periods, linear regression and non-linear regressions were used to find the best-fit relationship between mass loss $\%$ and the WES. To test the relationship between (cumulative or period) mass loss $\%$ in the termite access treatment and termite abundance at each harvest time, linear and non-linear regressions were used to find the best-fit relationship between mass loss \% and termites abundance. We used Student's t-test to test the differences in cumulative mass loss \% of termite access and exclusion treatment, termite abundance and the contribution of termites to wood mass loss between the sites.

To evaluate the relationship between termite abundance and WES in the two sites at each harvest time, linear and non-linear regressions were used to find the best-fit relationship. To evaluate the effect size of the termites along the WES of the two sites at each harvest time, linear and non-linear regressions were used to find the best-fit relationship. For mass loss data, we used Levene's test to examine the homogeneity of variance and Shapiro-Wilk test for normality. Wood mass loss was log-transformed as to best meet the assumptions of normality and variance homogeneity. All statistical analyses were performed in $R$ language version 3.5.1.

\section{RESULTS}

\section{Overall termite impact on wood decomposition}

Comparisons of the termite exclusion and access treatments strongly support the view that termites play an important role in coarse wood mass loss (Fig. 2), not only in tropical (see Introduction) but also in subtropical forests. Specifically, after 18 months of incubation of the wood samples, the termite access treatment resulted in an average cumulative mass loss of $26.6 \%$ (range 1.5-84.6\%) at PT and $21.1 \%$ (range 1.18-68.8\%) at TT across species. In contrast, mass loss without termite consumption was on average $14.7 \%$ (range 1.1-60.7\%) at PT and 12.7\% (range 1.0-45.7\%) at TT (Fig. S2a,b). The difference between termite exclusion and access treatments was generally high during the first 12 months and then decreased at 18 months in both sites (Fig. 2, Fig. S2a,b).

\section{Relationships between wood quality, termite activity and decomposition over time}

Multiple traits, notably wood N, P, cellulose and lignin content and wood density, together explained $55.9 \%$ of all variance explained by PC1, interpreted here as the WES (see Methods and Fig. S1). Wood litter of more resource acquisitive strategy species (i.e., high WES values), generally decompoesed faster than that of conservative strategy species; in the treatment without termite access (Fig. S1, Fig. 2) there were significant positive linear relationships between WES score and mass loss in the periods 0-6 and 12-18 months (but 
non-significant ones at 6-12 months), and cumulatively over 18 months, in both sites.

Our termite access treatment revealed that termites not only significantly accelerated wood mass loss rate overall, but there was a clear time pattern within this acceleration (Fig. 3), as hypothesized (Fig. 1). In the first year (periods 0-6, 6-12 months) decomposition rates scaled positively and linearly with WES in both sites (Fig. 2a,b,c,d), indicating that termites preferentially consumed the species of resource acquisitive strategy (high nutrient content, less lignin, less dense structure). Thereby, the termites' consumption amplified the initial WES effect on decomposability, i.e., it increased its positive linear regression slope.

In contrast, partly owing to termite activity, the wood of the acquisitive species had been considerably depleted after 12 months. In the subsequent period of 12-18 months, the initially medium quality species were consumed more by the termites, which modulated the tree species' decomposition trajectory on the WES. Now, in contrast to the positive linear relation in the treatment without termite access, there was a hump-back relationship between WES and period mass loss in the treatment with termite access, at least in the more termite-rich PT site (Fig. 2e, Table 1). Termite abundance showed a corresponding humpback pattern with mass loss (equation in Fig. 2e). In the less termite-rich site, TT, such a humpback relation was not apparent, but here the positive relationship between WES and mass loss was less steep in the latter (Fig. 2d,f) than in the initial period (Fig. 2b). This pattern also suggests negative termite feedback on the positive relation between WES and mass loss. These changing patterns of WES over period-specific mass loss were confirmed by a significant interaction among WES (covariate), harvest time and termite presence/absence on period mass loss in PT and TT, respectively (Table 2). Together, as hypothesized (Fig. 1), these deviating relationships over time caused overall convergence of cumulative mass loss along the WES between the termite treatments in both sites (Fig. 2g,h), as indicated by a lack of interaction of WES (covariate) x termite access treatment on cumulative mass loss over 18 months (Table 2).

Termite abundance patterns in the wood samples were consistent with the above changing patterns of periodspecific wood mass loss (Fig. 3) and the termite contribution to decomposition (Fig. S3) along the WES through time. At 6 months, there was a significant, exponential increase from the conservative end to the acquisitive end of the WES in both sites (Fig. 3a,b). At 12 months, the termite abundance peak occurred at slightly higher than medium initial wood quality (Fig. 3c,d). By 18 months in PT, the termite abundance peak had moved further towards the conservative end of the WES (Fig. 3e), i.e., to the centre of the range, matching the humpback for mass loss (Fig. 2e). In TT both the height and the width of the peak (i.e., the range) increased at 12-18 months (Fig. 3f). All in all, the peaks of termite abundance and termite contribution to mass loss shifted from high to medium initial wood quality during the decomposition process, broadly matching that of mass loss itself.

\section{Comparison of tree functional diversity as linked to animal abundance between sites}

We have strong albeit indirect evidence that the above linkages between initial deadwood quality, termites and decomposition through time had cascading effects, via termites populations, to predator (pangolin) populations, respectively. The CWM of WES (PC1) of deadwood species collected from PT was -0.02+0.03 (range -0.289 to 0.190 ) (PC1), while that from TT was -0.035+-0.04 (range -0.744 to 0.090) (Table 1); i.e., resource-acquisitive species were broadly more prevalent in PT and resource-conservative species more prevalent in TT. The higher mean WES value, and the higher functional diversity (i.e., abundance-weighted WES variance), corresponded with the higher termite abundance in PT than that in TT at the beginning of the experiment. After the wood samples (with overall range -3.99 to 4.65 along the WES) had been put in the plots, termite abundance showed an increasing trend in both sites, resulting in significantly higher values by approximately $250 \%$ after 18 months of incubation (Table 1 ). On the whole, the termite abundance in PT was about twice as high as that in TT (Table 1). In turn, in line with our expectations, pangolin mound density was significantly and four times higher in the experimental plots with deadwood samples added (thereby increasing termite abundance) compared to the control plots in PT (Table 1).

\section{DISCUSSION}

To our knowledge, this is the first study to experimentally unravel cascading effects of tree functional diversity, 
through trophic chains, on a biogeochemical process through time. Our concept and findings have added a new dimension to the "afterlife effects" of functional traits by pinpointing the cascading effects of variation in deadwood quality among tree species through a "brown" food chain, with important consequences for both forest carbon turnover and animal populations. While, without the involvement of invertebrate decomposers wood decomposition rates were predicted by initial values along the WES, when termites participated in the decomposition process they actively altered the species' ranking in terms of the WES through time and moved in abundance and activity from initially high WES species to initially medium WES species. These findings have an interesting parallel with a recent study that found a modulating effect of invertebrate consumption on leaf mass loss rankings along an axis of initial litter quality, i.e., the leaf economics spectrum, over time (Guo et al. 2019). However, in that study the modulation was due to a single outbreak by detritivorous moth larvae, while in this study the decomposing invertebrates (i.e., termites) were present in considerable abundance through time in the studied forests, but changed their relative abundance and contribution to decomposition among tree species as decomposition progressed. As a consequence, the temporally stable overall consumption of deadwood by termites should help to sustain deadwood turnover in a forest of high functional diversity. In turn, negative feedback by pangolins (Fig. 1) should contribute to net carbon sequestration via controlling the termite populations, but we could not carry out manipulation of pangolins to quantify this feedback.

Interactive effects of top-down and bottom-up forces in regulating food web structure have been shown (Faithfull et al. 2011; Leibold et al. 1997). However, in our system the positive bottom-up control of termite populations by deadwood seemed to strongly outweigh the negative top-down control by pangolins, as the termite population was still larger in PT with than in TT without pangolins. To overcome predation pressure, prey communities may show compensatory population growth via faster regeneration (Crawley 1992). This can provide an explanation why the PT termite population did not decline with a significant increase in predator population (evidenced by pangolin burrow density). In a functionally diverse forest, with the wood resource complementarity as shown in our study, the basal resource constraint is likely relatively weak through time, allowing prey (termites) to reach high population density in spite of predator (pangolin) control. If hunting and trafficking of pangolins ceased completely (e.g., in response to pangolins' suspected role in transmission of the 2020 Covid-19 virus to humans), and pangolin populations were allowed to regain their natural population size across the entire region, the contributions of top-down versus bottom-up control of termite populations and their effect on wood decomposition and the forest carbon budget would be easier to study.

New experiments are needed to enrich our conceptual model with empirical evidence, beyond the specific food chain studied here. On the one hand, other termite predators in the world (e.g., other pangolin species; aardvarks in Africa; some armadillo species in the Americas) are also in dramatic population decline. We need to know whether, besides halting their persecution by people, improving their habitat by promoting plant functional diversity and, thereby, their staple food will help to recover or sustain the populations of these species in general. Some ants are also known to have termites as a staple foods (Buczkowski \& Bennett 2007), but whether or how they compete for termites with vertebrate termite feeders such as pangolins, or are themselves eaten and thereby controlled top-down by these vertebrates, and thereby indirectly affect wood decomposition, are intriguing questions for further investigation.

Moreover, terrestrial decomposition is integrated in multiple food chains in the food web. Thus, to better understand the generality of the positive deadwood turnover feedback studied here, we need to identify the interactions between other wood (or leaf, root) decomposing invertebrates or predators of other detritivores and plant functional trait diversity. Although termites are the dominant species in warm-climate wood decomposition, other invertebrates such as beetles tend to control the animal contribution to decomposition in temperate ecosystems; either directly through feeding or by facilitating other decomposers such as fungi or similar-sized animals to invade the deadwood by tunneling, feeding, nesting or intended microbe cultivation (Ulysen 2016; Zuo et al. 2014). In our experiment we found a wide variety of deadwood beetles and millipedes; although their abundance is far less than that of termites, how (much) they affect wood decomposition, directly or by affecting other decomposers such as fungi and termites, needs in-depth study. Importantly, 
we need to study how these different decomposers, through time (e.g., decay stages, seasons), interact with each other and with the trait diversity of their basal resource via their decomposing function. Multiple harvests and tracking animal population dynamics (Schwarzmuller et al. 2015) in such experiments can provide further insights into time effects of decomposers, and their suppression by predators, as influenced by resource availability.

Taken together, our findings strongly point to high wood functional diversity helping to provision sufficient food resource in terms of deadwood quantity and quality for termites to maintain their population through time. In turn, this larger and stable termite population seemed to have increased the density of pangolins as indicated by the four-fold increase in density of their burrows. Thus, our results strongly suggest that woody plant functional (trait) diversity can support a (threatened) mammal species at the top of the food chain through the detritivore subsystem. We have to make the caveat that the causality of wood functional diversity supporting the pangolin population in our experiment cannot be proven and would need further, perhaps manipulative study. The fact that we added deadwood in our research plots may by itself have stimulated both termites and pangolins and, in theory, year-to-year changes in environmental conditions could also have interfered with termite and pangolin populations. Still, our results strongly suggest that, if only initially high, medium or low quality wood had been added in the experiment, the termite populations would not have increased as much as they did with the broader resource economic spectrum (also compared to the initial forest community) incubated in our forest plots over the time span of the experiment. And neither would the number of pangolin burrows in the PT site have quadrupled. Moreover, although indirect evidence, the (living) tree community in PT, with the larger overall termite density, had a greater wood trait diversity than in TT (Table 1, unweighted WES range, community-weighted WES variance), which likely translates into a wider overall range in deadwood quality in PT. Thus, our results are consistent with the hypothesis that woody plant functional (trait) diversity drives an intriguing interplay of dead wood quality, its decomposition and termites through time, thereby even supporting a threatened mammal at the top of the food chain. These findings should invite further studies, in other green or brown food webs, of how plant functional diversity can cascade dynamically through different trophic levels all the way to the top of food chains.

\section{ACKNOWLEDGMENTS}

The authors would like to thank Minshan Xu, Liting Zheng, Xiangyu Liu, Umar Aftab Abbasi, Tian Su, Wujian Xiong, Qiming Liang, Xiaotong Zhu, Liang Li, Dong He and Liangyan Wang for their assistance preparing the wood samples in the field and laboratory. This study was supported by the National Natural Science Foundation of China (Grant Nos. 31770467 and 31670438). The authors declare no competing interests.

\section{REFERENCES}

Balvanera, P., Pfisterer, A. B., Buchmann, N., He, J. S., Nakashizuka, T., Raffaelli, D., \& Schmid, B. (2006). Quantifying the evidence for biodiversity effects on ecosystem functioning and services. Ecol. Lett. , 9, 1146-1156.

Baraloto, C., Timothy Paine, C. E., Poorter, L., Beauchene, J., Bonal, D., Domenach, A. M., et al . (2010). Decoupled leaf and stem economics in rain forest trees.Ecol. Lett., 13, 1338-1347.

Bascompte, J., Jordano, P., Melian, C. J., \& Olesen, J. M. (2003). The nested assembly of plant-animal mutualistic networks. Proc. Natl. Acad. Sci. U. S. A. , 100, 9383-9387.

Borenstein, M., Hedges, L. V., Higgins, J. P., \& Rothstein, H. R. (2009). Introduction to meta-analysis . John Wiley \& Sons.

Buczkowski, G., \& Bennett, G. (2007). Protein marking reveals predation on termites by the woodland ant, Aphaenogaster rudis. Insect. Soc. , 54, 219-224.

Corlett, R. T. (2007). The impact of hunting on the mammalian fauna of tropical Asian forests. Biotropica 
, 39, 292-303.

Cornelissen, J.H.C., Sass-klaassen, U., Poorter, L., van Geffen, K., van Logtestijn, R.S.P., van Hal, J. et al . (2012). Controls on coarse wood decay in temperate tree species: birth of the LOGLIFE experiment.Ambio , 41, 231-245.

Crawley, M. J. (1992). In Natural Enemies: The Population Biology of Predators, Parasites and Diseases.Blackwell Scientific Publications, 40-90.

Diaz, S., Lavorel, S., de Bello, F., Quetier, F., Grigulis, K., \& Robson, T. M. (2007). Incorporating plant functional diversity effects in ecosystem service assessments. Proc. Natl. Acad. Sci. U. S. A., 104, 20684-20689.

Fargione, J., Tilman, D., Dybzinski, R., Lambers, J. H. R., Clark, C., Harpole, W. S., et al . (2007). From selection to complementarity: shifts in the causes of biodiversity-productivity relationships in a long-term biodiversity experiment. Proc. R. Soc. B-Biol. Sci. , 274, 871-876.

Faithfull, C. L., Huss, M., Vrede, T., \& Bergstrom, A. K. (2011). Bottom-up carbon subsidies and top-down predation pressure interact to affect aquatic food web structure. Oikos , 120, 311-320.

Freschet, G.T., Aerts, R. \& Cornelissen, J.H.C. (2012). A plant economics spectrum of litter decomposability. Funct. Ecol. , 26, 56-65.

Guo, C., Cornelissen, J. H. C., Tuo, B., Ci, H., \& Yan, E. R. (2019). Invertebrate phenology modulates the effect of the leaf economics spectrum on litter decomposition rate across 41 subtropical woody plant species. Funct. Ecol. , 34, 735-746.

Guo, C., Cornelissen, J. H. C., Bin, T., Ci, H. \& Yan, E. R. (2020). Data from: Dynamic feedbacks among tree species traits, termites and an endangered mammal via deadwood turnover. Dryad, Dataset .

Hattenschwiler, S., Tiunov, A. V. \& Scheu, S. (2005). Biodiversity and litter decomposition in terrestrial ecosystem. Annu. Rev. Ecol. Evol. Syst. , 36, 191-218.

Handa, I. T., Aerts, R., Berendse, F., Berg, M. P., Bruder, A., Butenschoen, O., et al. (2014). Consequences of biodiversity loss for litter decomposition across biomes. Nature, 509, 218.

Haddad, N. M., Crutsinger, G. M., Gross, K., Haarstad, J., Knops, J. M., \& Tilman, D. (2009). Plant species loss decreases arthropod diversity and shifts in trophic structure. Ecol. Lett. , 12, 1029-1039.

Haddad, N. M., Crutsinger, G. M., Gross, K., Haarstad, J., \& Tilman, D. (2011). Plant diversity and the stability of foodwebs. Ecol. Lett. , 14, 42-46.

Hector, A., Schmid, B., Beierkuhnlein, C., Caldeira, M. C., Diemer, M., Dimitrakopoulos, P. G., et al . (1999). Plant diversity and productivity experiments in European grasslands. Science, 286, 1123-1127.

Hooper, D. U., \& Dukes, J. S. (2004). Overyielding among plant functional groups in a long-term experiment. Ecol. Lett., 7, 95-105.

Hooper, D. U., \& Vitousek, P. M. (1998). Effects of plant composition and diversity on nutrient cycling. Ecol. Monogr., 68, 121-149.

IPBES. (2019). Global assessment report on biodiversity and ecosystem services of the Intergovernmental Science- Policy Platform on Biodiversity and Ecosystem Services. IPBES, IPBES Secretariat, Bonn, Germany. https://www.ipbes.net/global-assessment-report-biodiversity-ecosystemservices.

IUCN. (2016). The IUCN Red List of Threatened Species. Version 2016-3. Available at: www.iucnredlist.org.

Jones, D. T., \& Eggleton, P. (2000). Sampling termite assemblages in tropical forests: testing a rapid biodiversity assessment protocol.J. Appl. Ecol. , 37, 191-203. 
Jones, C.G., Lawton, J.H. \& Shachak, M. (1997). Positive and negative effects of organisms as physical ecosystem engineers. Ecology,78, 1946-1957.

Leibold, M. A., Chase, J. M., Shurin, J. B., \& Downing, A. L. (1997). Species turnover and the regulation of trophic structure. Ann. Rev. Ecol. Syst. , 28, 467-494.

Liu, G., Cornwell, W. K., Cao, K., Hu, Y., Van Logtestijn, R. S., Yang, S., et al . (2015). Termites amplify the effects of wood traits on decomposition rates among multiple bamboo and dicot woody species.J. Ecol. , 103, 1214-1223.

McMeans, B. C., McCann, K. S., Humphries, M., Rooney, N., \& Fisk, A. T. (2015). Food web structure in temporally-forced ecosystems. Trends Ecol. Evol. , 30, 662-672.

Poorter, H. \& Villar, R. (1997). The fate of acquired carbon in plants: chemical composition and construction costs. Plant Resource Allocation (eds F.A. Bazzaz andJ. Grace), Academic Press, SanDiego, CA, pp. 39-72.

Pietsch, K. A., Ogle, K., Cornelissen, J. H. C., Cornwell, W. K., Bonisch, G., Craine, J. M., et al . (2014). Global relationship of wood and leaf litter decomposability: the role of functional traits within and across plant organs. Glob. Ecol. Biogeogr. , 23, 1046-1057.

Scherber, C., Eisenhauer, N., Weisser, W. W., Schmid, B., Voigt, W., Fischer, M., et al . (2010). Bottom-up effects of plant diversity on multitrophic interactions in a biodiversity experiment.Nature , 468, 553-556.

Schwarzmuller, F., Eisenhauer, N., \& Brose, U. (2015). 'Trophic whales' as biotic buffers: weak interactions stabilize ecosystems against nutrient enrichment. J. Anim. Ecol. , 84, 680-691.

Srivastava, D. S., Cardinale, B. J., Downing, A. L., Duffy, J. E., Jouseau, C., Sankaran, M., et al . (2009). Diversity has stronger top-down than bottom-up effects on decomposition. Ecology, 90, 1073-1083.

Takamura, K. (2001). Effects of termite exclusion on decay of heavy and light hardwood in a tropical rain forest of Peninsular Malaysia.J. Trop. Ecol. , 17, 541-548.

Thakur, M. P., \& Eisenhauer, N. (2015). Plant community composition determines the strength of top-down control in a soil food web motif.Sci. Rep. , 5, 9134.

Ulyshen, M. D. (2016). Wood decomposition as influenced by invertebrates. Biol. Rev. , 91, 70-85.

Violle, C., Navas, M. L., Vile, D., Kazakou, E., Fortunel, C., Hummel, I., et al . (2007). Let the concept of trait be functional.Oikos, 116, 882-892.

Voigt, W., Perner, J., Davis, A. J., Eggers, T., Schumacher, J., Bahrmann, R., et al . (2003). Trophic levels are differentially sensitive to climate. Ecology , 84, 2444-2453.

Williamson, G. B., \& Wiemann, M. C. (2010). Measuring wood specific gravity correctly. Am. J. Bot. , 97, 519-524.

Wood, T.G. \& Sands, W.A. (1978). The role of termites in ecosystems. Production Ecology of Ants and Termites (ed. M.V. Brian) . Cambridge University Press, pp. 245-292.

Wu, S. B., \& Ma, G. Z. (2007). The status and conservation of pangolins in China. TRAFFIC East Asia Newsletter , 4, 1-5.

Yan, E. R., Wang, X. H., \& Huang, J. J. (2006). Shifts in plant nutrient use strategies under secondary forest succession. Plant Soil , 289, 187-197.

Yi, L., You, W. \& Song, Y. (2006). Soil animal communities at five succession stages in the litter of the evergreen broad-leaved forest in Tiantong, China. Frontiers of Biology in China, 1, 142-150.

Zuo, J., Fonck, M., van Hal, J., Cornelissen, J. H. C., \& Berg, M. P. (2014). Diversity of macro-detritivores in dead wood is influenced by tree species, decay stage and environment. Soil Biol. Biochem. , 78, 288-297. 
Zuo, J., Hefting, M. M., Berg, M. P., van Logtestijn, R. S., van Hal, J., Goudzwaard, L., et al . (2018). Is there a tree economics spectrum of decomposability? Soil Biol. Biochem. , 119, 135-142.

\section{Figure Legends}

Figure 1 Conceptual model hypothesizing the feedbacks between tree functional diversity, maintenance of termite populations, dead wood (carbon) turnover and mammal predator (here pangolin) populations. Wood litters of a wide range of functionally different tree species in terms of their position along the wood economics spectrum (WES) enter the forest floor as dead wood owing to logging or typhoon impact (Time 0). At time 1, during the initial period of decay, the termites prefer tree species with high dead wood quality (i.e., high WES values), where they reach high abundance and accelerate the consumption and carbon turnover of high-quality wood. After the high quality wood has mostly been used up (Time 2), the medium quality wood has decayed somewhat and become more palatable; thus, the initially medium quality wood should now have higher termite abundance and consumption leading to relatively high decomposition rate. Thus, with high tree functional diversity there should be stable availability of dead wood of suitable quality on the forest floor to maintain termite populations, thereby also promoting stable prey availability to sustain the pangolin population. If and where pangolins are lost, and thereby the "top-down" control over termite populations, the latter will only be controlled "bottom-up" and dead wood turnover will increase.

Figure 2 Relationships between the wood economics spectrum (PC1 values) and wood mass loss with termite access (open circles, solid line) and without termite access (filled circles, dotted line) in the two sites (Putuo versus Tiantong) at each harvest time (period mass loss) and cumulatively over the entire 18 months. The best-fit regression lines with confidence intervals (between dotted lines) are given where significant. The size (diameter) of the circles denotes termite abundance, and the colour gradient from blue to red for the circles denotes the conservative end to the acquisitive end of the WES. Regression equations are also given for the relationships between termite abundance and mass loss in the dead wood samples across species. The comparison of overall final cumulative mass loss between Putuo and Tiantong with and without termite access is shown in the inserted bar diagrams. ${ }^{* * *}, \mathrm{P}<0.001$; ns, not significant.

Figure 3 Relationships between the WES values (PC1) and termite abundance in the two respective sites across harvest times. The best-fit regression lines with confidence intervals (between dotted lines) are given where significant. The colour gradient from blue to red colour for the circles denotes the conservative end to the acquisitive end of the WES. The comparison of overall termite abundance between Putuo and Tiantong with and without termite access is shown in the inserted bar diagrams. ${ }^{* * *}, \mathrm{P}<0.001$; ns, not significant.

\section{Figure 1}




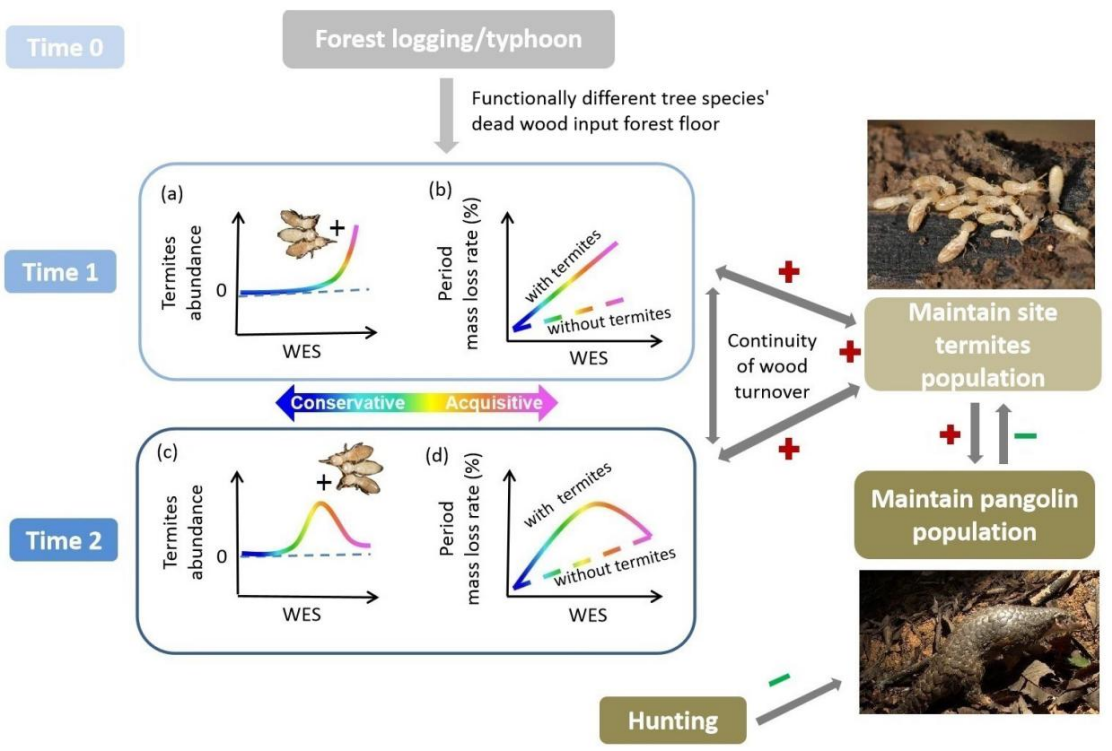

Figure 2 

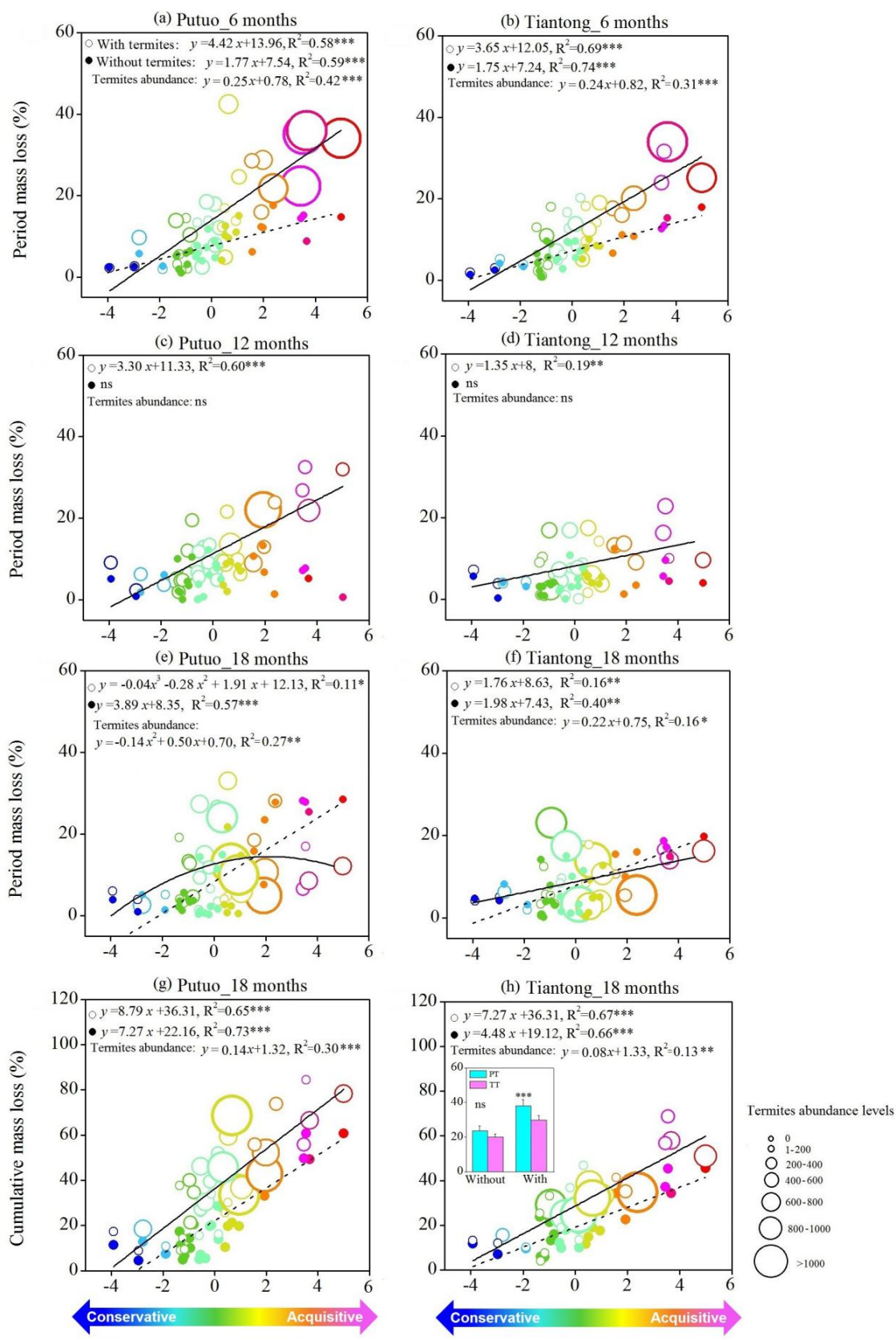

Wood economics axis (PCl)

Figure 3 

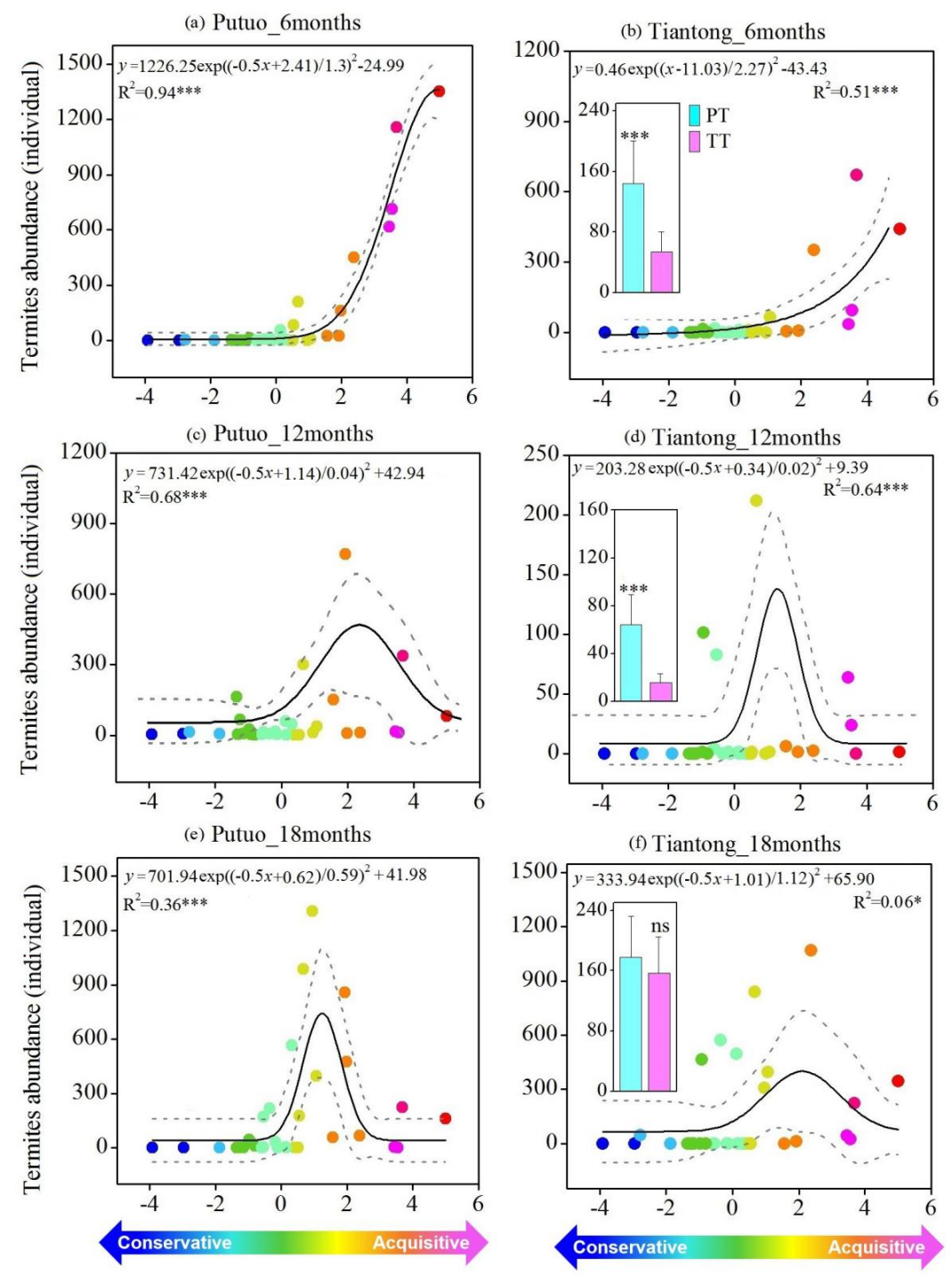

Wood economics axis (PC1)

Table

Table 1. Comparison of the biotic and soil characteristics of the two sites where the wood samples were incubated

\section{Variables}

Forest type

Mean annual temperature

Mean annual precipitation

Dominant species

Range of the community wood economic spectrum (PC1)

CWM of wood economic spectrum (PC1)

CWM of specific leaf area

CWM of wood density

\section{Putuo (PT)}

Evergreen broad-leaved forest

$16{ }^{\circ} \mathrm{C}$

$1358 \mathrm{~mm}$

Quercus glauca

$-2.06-3.44$

$-0.02 \pm 0.03$

$122.72 \pm 6.74 \mathrm{NS}$

$0.64 \pm 0.03 \mathrm{NS}$
Tianton

Evergre

$16.2{ }^{\circ} \mathrm{C}$

$1375 \mathrm{ml}$

Schima

$-2.94-2$

$-0.035 \pm$

$109.40 \pm$

$0.56 \pm 0$. 
Soil type

Soil pH

Soil available $\mathrm{K}\left(\mathrm{mg} \cdot \mathrm{kg}^{-1}\right)$

Soil available $\mathrm{P}\left(\mathrm{g} \cdot \mathrm{kg}^{-1}\right)$

Soil organic matter $\left(\mathrm{g} \cdot \mathrm{kg}^{-1}\right)$

Soil N $\left(\mathrm{g} \cdot \mathrm{kg}^{-1}\right)$

Termite density (ind $\cdot \mathrm{m}^{-2}$ ) in Aug. 2018

Termite density (ind $\cdot \mathrm{m}^{-2}$ ) in Aug. 2019

Pangolin burrow density (ind $\cdot \mathrm{ha}^{-1}$ ) in wood sample incubated plots in Nov. 2019

Pangolin burrow density (ind $\cdot \mathrm{ha}^{-1}$ ) in control plots in Nov. 2019
Sandy clay laterite

$4.78 \pm 0.03 \mathrm{NS}$

$14.76 \pm 0.75 \mathrm{NS}$

$5.19 \pm 0.53 *$

$6.70 \pm 0.58 * *$

$1.06 \pm 0.05 *$

$153.23 \pm 20.92$ *

$395.22 \pm 38.95^{* *}$

$133 \pm 30 * * *$

$33 \pm 8 * * *$
Red anc $4.09 \pm 0$. $16.65 \pm 1$ $2.33 \pm 0$. $20.43 \pm 0$ $3.44 \pm 0$. $67.18 \pm 1$ $184.20 \pm$ 0 0

Note: Asterisk indicated there was significant difference between TT and PT; ${ }^{*} p<0.05 ;{ }^{* *} p<0.01 ;{ }^{* * *} p$ $<0.001 ;$ NS, No significant difference.

Table 2. Results of Analysis of covariance (with WES as the covariate) testing the interactive effects between harvest time and termite presence/absence on the period wood mass loss and cumulative wood mass loss over 18 months in PT and TT.

\begin{tabular}{|c|c|c|c|c|c|}
\hline & & Putuo (PT) & Putuo (PT) & Tiantong (TT) & Tiantong (TT) \\
\hline Period wood mass loss & Df & $\mathrm{F}$ & $\mathrm{P}$ & $\mathrm{F}$ & $\mathrm{P}$ \\
\hline Harvest time & 2 & 3.41 & $<0.05$ & 9.62 & $<0.001$ \\
\hline Termite presence & 1 & 39.18 & $<0.001$ & 19.17 & $<0.001$ \\
\hline WES & 1 & 99.89 & $<0.001$ & 93.50 & $<0.001$ \\
\hline Harvest time $\times$ termite presence & 2 & 1.58 & 0.21 & 0.84 & 0.44 \\
\hline Harvest time $\times$ WES & 2 & 3.29 & $<0.05$ & 4.30 & $<0.05$ \\
\hline Termite presence $\times$ WES & 1 & 0.10 & 0.75 & 0.33 & 0.56 \\
\hline Harvest time $\times$ termite presence $\times$ WES & 5 & 10.13 & $<0.001$ & 2.90 & $<0.05$ \\
\hline Residuals & 206 & & & & \\
\hline \multicolumn{6}{|l|}{ Cumulative wood mass loss } \\
\hline Termite presence & 1 & 34.45 & $<0.001$ & 37.68 & $<0.001$ \\
\hline WES & 1 & 130.85 & $<0.001$ & 210.26 & $<0.001$ \\
\hline Termite presence $\times \mathrm{WES}$ & 2 & 0.86 & 0.35 & 1.71 & 0.19 \\
\hline Residuals & 206 & & & & \\
\hline
\end{tabular}

Note: Df: Degree of freedom 